\title{
Establishment of the monomeric yellow-green fluorescent protein mNeonGreen for life cell imaging in mycelial fungi
}

\author{
Antonia Werner, Kolja L. Otte, Gertrud Stahlhut and Stefanie Pöggeler *i]
}

\begin{abstract}
The engineered monomeric version of the lancelet Branchiostoma lanceolatum fluorescent protein, $\mathrm{mNeonGreen}$ $(\mathrm{mNG})$, has several positive characteristics, such as a very bright fluorescence, high photostability and fast maturation. These features make it a good candidate for the utilization as fluorescent tool for cell biology and biochemical applications in filamentous fungi. We report the generation of plasmids for the expression of the heterologous mNG gene under the control of an inducible and a constitutive promoter in the filamentous ascomycete Sordaria macrospora and display a stable expression of $\mathrm{mNG}$ in the cytoplasm. To demonstrate its usefulness for labeling of organelles, the peroxisomal targeting sequence serine-lysine-leucine (SKL) was fused to $\mathrm{mNG}$. Expression of this tagged version led to protein import of $\mathrm{mNG}$ into peroxisomes and their bright fluorescence in life cell imaging.
\end{abstract}

Keywords: mNeonGreen, Sordaria macrospora, Peroxisomes, Fluorescent protein

\section{Introduction}

Fluorescence microscopy is a potent method for studying in vivo protein localization, cytoskeleton dynamics, infections and protein-protein-interactions in filamentous fungi (Berepiki et al. 2010; Chapuis et al. 2019; Hoff and Kück 2005; Lorang et al. 2001).

Fluorescent proteins can be expressed in fungal cells by inserting genes encoding fluorescent proteins into the genome under the control of constitutive or inducible promoters (Oda et al. 2016; Pöggeler et al. 2003). The green fluorescent protein GFP from the jellyfish Aequorea victoria and its engineered versions with altered spectral properties, such as yellow and cyan-shifted mutants, enhanced brightness versions or $\mathrm{pH}$-sensitive variants have been utilized in fungal cell biology for many years (Bagar et al. 2009; Chalfie et al. 1994; Chudakov et al. 2005; Lorang et al. 2001; Rech et al. 2007).

\footnotetext{
*Correspondence: spoegge@gwdg.de

Department of Genetics of Eukaryotic Microorganisms, Institute

of Microbiology and Genetics, Georg-August-University of Göttingen,

Grisebachstr. 8, 37077 Göttingen, Germany
}

In addition, the tetrameric fluorescent protein DsRed, its monomeric variant mRFP from the marine anemone Discosoma striata as well as brighter versions of mRFP (mCherry and tdTomato) emitting in the red light spectrum have been successfully expressed in filamentous fungi (Campbell et al. 2002; Mikkelsen et al. 2003; Schuster et al. 2015; Toews et al. 2004; Xiao et al. 2018). Furthermore, the red fluorescent TagRFP-T, from the sea anemone Entacmaea quadricolor has been applied in fungal life cell imaging (Schuster et al. 2015).

Recently, the new and very bright yellow fluorescent protein LanYFP with a high quantum yield $(\sim 0.95)$ and extinction coefficient $\left(\sim 150,000 \mathrm{M}-1 \mathrm{~cm}^{-1}\right)$ was isolated from lancelet Branchiostoma lanceolatum. Engineering of the tetrameric LanYFP resulted in a $26.5 \mathrm{kDa}$ monomeric, yellow-green fluorescent protein called mNeonGreen (mNG) (Shaner et al. 2013). In comparison to LanYFP, mNG harbors 21 substitutions and contains modified $\mathrm{N}$ - and $\mathrm{C}$-termini of additional 11 and 7 amino acids derived from eGFP. mNG has a blue-shifted excitation maximum of $506 \mathrm{~nm}$ and an emission maximum of $517 \mathrm{~nm}$ with a high quantum yield excitation coefficient. 
Compared to eGFP it has a nearly threefold brighter emission, an increased photostability and shorter maturation time (Shaner et al. 2013). The new yellow- green fluorescent protein has been used mainly for fluorescent microscopy. It has been applied as genetically encoded tag to visualize gene products and cellular compartments in bacteria, animals, plants and yeast (Botman et al. 2019; Heppert et al. 2016; Hostettler et al. 2017; Stoddard and Rolland 2019; Wilton et al. 2017). Only one report exists on expression mNG in filamentous fungi. Recently, in Aspergillus fumigatus a version of mNG codon-optimized for the green algae Chlamydomonas rheinhardii was shown to be expressed under control of the strong constitutive tefA promoter (Pham et al. 2020).

Often expression of fluorescent proteins in filamentous fungi requires species-specific variants that can be efficiently transcribed and translated in the respected host. Therefore, often codon-optimization of the coding region and a fungal promoter that meets the conditions for a specific experimental set-ups have to be established (Lorang et al. 2001).

In this study, we demonstrate the successful expression of $\mathrm{mNG}$ in the filamentous ascomycete Sordaria macrospora, a model organism for investigating fruiting body development and meiosis in fungi (Teichert et al. 2014, 2020; Zickler and Espagne, 2016). We generated plasmids for expression of mng under the control of the constitutive gpd promoter of Aspergillus nidulans and the xylose inducible $S m x y l$ promoter of S. macrospora (Bloemendal et al. 2014). To demonstrate the usefulness of mNG for visualization of fungal organelles, we fused the peroxisomal localization signal SKL to $\mathrm{mNG}$ and monitored peroxisomes in S. macrospora.

\section{Material and methods \\ Strains, media and growth conditions}

Escherichia coli strain MACH1 (Thermo Fisher Scientific, C862003, Waltham, USA) was used for cloning and propagation of recombinant plasmids using standard culture conditions (Sambrook et al. 2001). Homologous recombination was performed to generate recombinant plasmids in Saccharomyces cerevisiae strain PJ694A (Colot et al. 2006; James et al. 1996). S. macrospora strains were transformed with recombinant plasmids as described previously (Walz and Kück 1995). Selection of transformants was performed on media containing nourseothricin-dihydrogen sulphate $\left(50 \mu \mathrm{g} \mathrm{ml}{ }^{-1}\right)$ (Jena Bioscience GmBH, AB-102XL, Jena, Germany). S. macrospora strains were grown at $27^{\circ} \mathrm{C}$ on liquid or solid biomalt maize medium (BMM), or Sordaria Westergaard (SWG) fructification medium under continuous light conditions (Elleuche and Pöggeler 2008; Esser 1982; Nowrousian et al. 2005). To induce the xylose promoter, SWG medium was prepared with $2 \%$ xylose replacing glucose.

\section{Generation of plasmids}

All primers and template plasmids used for PCR amplifications are listed in Tables 1 and 2, respectively. Primers

Table 1 List of primers used in this study

\begin{tabular}{|c|c|}
\hline Name & Sequence $\left(5^{\prime}-3^{\prime}\right)$ \\
\hline Pxyl_fw & GACTGGTCTCAAGTC $_{\text {GAACCTTCTCTTCTCCATTTCTT }}$ \\
\hline Pxyl_rev & CAGAGGTCTCAGCAG GTTGGCGGTTTCTGGTTAGGCC \\
\hline Neon_fw & CAGAGGTCTCACTGC ATGGTGAGCAAGGGCGAGGAGG \\
\hline Neon_rev & CTCAGGTCTCCCGTA CTACTTGTACAGCTCGTCCATGC \\
\hline Pxylneon_fw & GTAACGCCAGGGTTTTCCCAGTCACGACG GAACCTTCTCTTCTCCATTT \\
\hline Pxylneon_rev & 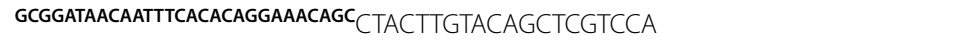 \\
\hline trpC-neon & GCCTTTACCGATGTGATGGGCATGGACGAGCTTTACAAGTAG AGCGGCCGCTCTAGAACTAGTGGATCCACT \\
\hline TtrpC_pRS_r & GCGGATAACAATTTCACACAGGAAACAGC TCGAGTGGAGATGTGGAGTGG \\
\hline Neon_fw-2 & cGAGTCAGGTCTCCTGGT ATGGTGAGCAAGGGCGAG \\
\hline & GAGG \\
\hline Neon_rev-2 & ACCGACAGGTCTCGATCC CTTGTACAGCTCGTCCATGC \\
\hline Neo_f & ATGGTGAGCAAGGGCGAG \\
\hline Neo_SKL_TrpC_r & GTTTGATGATTTCAGTAACGTTAAGTGGA $T T A G A G C T T G C T C T T G T A C A G C T C G T C C A T G C$ \\
\hline pRS_Pgpd_f & 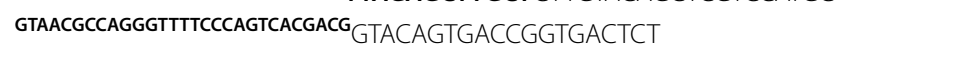 \\
\hline Pgpd_Neo_r & ATGTTATCCTCCTCGCCCTTGCTCACCAT GGGAAAAGAAAGAGAAAAGAAAAGAGCA \\
\hline TtrpC_f & TCCACTTAACGTTACTGAAATCATCAAAC \\
\hline
\end{tabular}

bold superscript $=$ overhang; superscript underlined = the four base pairs after the Bsal recognition site in the overhang, bold italics = tripeptide SKL and a stop codon $T$ terminator, $P$ promotor 
Table 2 List of plasmids used in this study

\begin{tabular}{|c|c|c|}
\hline Plasmid & Characteristics & Source \\
\hline pRS-nat & $a m p^{R}, u r a 3, n a t^{R}$ & (Klix et al. 2010) \\
\hline p1783-1 & $\begin{array}{l}\text { amp }^{R}, \text { ura3 }, \text { hyg }^{R} \\
\text { Pgpd::egfp::TtrpC }\end{array}$ & (Pöggeler et al. 2003) \\
\hline pRHN1 & $\begin{array}{l}\text { amp }^{R}, \text { ura3 }, \text { nat }^{R} \\
\text { Pgpd::Dsred::Ttrpc }\end{array}$ & (Janus et al. 2007) \\
\hline pDsRed-SKL & $\begin{array}{l}\text { amp }^{R}, \text { nat }^{R} \\
\text { Pgpd::Dsred-SKL:::TtrpC }\end{array}$ & $\begin{array}{l}\text { (Elleuche and Pöggeler } \\
\text { 2008) }\end{array}$ \\
\hline pDest::amp & $a m p^{R}$ & Dahlmann et al. 2020 \\
\hline $\mathrm{pGG}-\mathrm{C}-\mathrm{F}$ & $\begin{array}{l}\text { amp }^{R}{ }_{\text {"nat }}{ }^{R} \\
\text { Pgpd::3xFLAG::TtrpC }\end{array}$ & Dahlmann et al. 2020 \\
\hline pNpX-GFP & $\begin{array}{l}\text { amp }^{R}, \text { ura3 }, \text { nat }^{R} \\
\text { Pgpd::egfp::TtrpC }\end{array}$ & (Bloemendal et al. 2014) \\
\hline $\begin{array}{l}\text { 753-pENTR20_- } \\
\text { mNeonGreen- } \\
\text { N1 }\end{array}$ & $k a n^{R}, m n g$ (optimized) & $\begin{array}{l}\text { Schink, pers. communica- } \\
\text { tion }\end{array}$ \\
\hline pxyl-mNeonGreen & $\begin{array}{l}\text { amp } p^{R}, \text { ura3, } n a t^{R} \\
\text { Pxyl::mng }\end{array}$ & This study \\
\hline pxyl-mng & $\begin{array}{l}a_{m p}^{R}, \text { ura3 }, \text { nat }^{R} \\
\text { Pxyl::mng::TtrpC }\end{array}$ & This study \\
\hline pGG-C-F-mng & $\begin{array}{l}\text { amp }{ }^{R}, \text { nat }^{R} \\
\text { Pgpd::mng::3xFLAG::TtrpC }\end{array}$ & This study \\
\hline pmng-SKL & $\begin{array}{l}\text { amp }{ }^{R}, \text { ura3, }_{\text {nat }}{ }^{R} \\
\text { Pgpd::mng-SKL::TtrpC }\end{array}$ & This study \\
\hline
\end{tabular}

nat ${ }^{R}$ : nourseothricin resistant, $h y g^{R}$ : hygromycin resistant; $a m p^{R}$ : ampicillin resistance; ura3, Orotidine-5'-phosphate decarboxylase gene of S. cerevisiae; Pxyl: promoter of the xylose gene Smxyl of Sordaria macrospora; Pgpd: promoter of the glycerinaldehyd-3-phosphat-dehydrogenase-gene of Aspergillus nidulans; TtrpC: terminator of the anthranilat synthase gene of Aspergillus nidulans; ssi: single spore isolate; SKL: peroxisomal targeting sequence Ser-Arg-Leu; $m n g$ : gene for green fluorescence protein monomeric NeonGreen, $(\mathrm{mNG})$ of Branchiostoma lanceolatum; Dsred: gene for red fluorescence protein (DsRed) of Discosoma species; egfp: gene for green fluorescence protein enhanced green fluorescent protein (eGFP) of Aequorea victoria

were synthesized by Sigma-Aldrich Chemie $\mathrm{GmbH}$ (Taufkirchen, Germany).

To generate a plasmid in which $\mathrm{mNG}$ is under control of an inducible promoter we used the Golden Gate cloning system (Terfrüchte et al. 2014). This system uses restriction enzyme $B s a \mathrm{I}$ hydrolyzing 4 base pairs after its recognition site, independent from the identity of these four base pairs, which allows the generation of sticky overhangs, which can be used for complex fragment assemblies (Dahlmann et al. 2020). For the PCR amplification of the $S$. macrospora $x y l$ promoter $S m x y l$, plasmid pNpX-GFP (Bloemendal et al. 2014) was used as template and Pxyl_fw and Pxyl_rev as primers. To amplify the gene coding for mNG, plasmid 753-pENTR20_mNeonGreen-N1 (a kind gift from Kay Oliver Schink, Centre for Cancer Biomedicine (CCB) Oslo University Hospital, Radiumhospitalet) served as template for PCR with primer pair Neon_fw/Neon_rev. The Smxyl-promoter fragment and the mng fragment were combined with vector pDest::amp (Dahlmann et al. 2020) by treatment with BsaI and T4 DNA-ligase. The resulting vector was used to amplify the Pxyl-mng fragment with the primer pair Pxylneon_fw/Pxylneon_rev. Both primers generated a 29-bp overhang at the PCR product which was together with XhoI linearized vector pRS-nat used for homologous recombination cloning in Saccharomyces cerevisiae strain PJ69-4A (Colot et al. 2006). Since the resulting plasmid pxyl-mNeonGreen revealed no fluorescence after transformation into S. macrospora, we decided to introduce the terminator sequence of the $\operatorname{trpC}$ gene of Aspergillus nidulans downstream to the mng coding sequence. Plasmid pxyl-mNeonGreen was linearized with BsRGI and the $A$. nidulans trp $C$ terminator was amplified with primer pair trpC-neon/TtrpC_pRS_r. Homologous recombination in $S$. cerevisiae resulted in plasmid pxylmng (Fig. 1a). To express mNG under the constitutive gpd promoter of $A$. nidulans, we utilized the Golden Gate cloning vector pGG-C-F. This vector contains a $3 \times$ FLAG tag flanked by the gpd promoter and the $\operatorname{trp} C$ terminator of $A$. nidulans (Teichert et. al. unpublished). The mng ORF without STOP codon was PCR amplified with the primer pair Neon_fw-2/Neon_rev-2 and 753pENTR20_mNeonGreen-N1 served as template. The resulting PCR fragment was combined with vector pGGC-F. Incubation with BsaI and T4 DNA-ligase resulted in plasmid pGG-C-F-mng (Fig. 1b).

To label peroxisomes with $\mathrm{mNG}$, we fused the peroxisomal targeting sequence 1 (PTS1) encoding the three amino acids serine-lysine-leucine (SKL) to the $\mathrm{C}$-terminus of the mng ORF. The coding sequence of the mng gene was amplified from plasmid 753_pENTR20_ mng-N1 with primer pair Neo_f/Neo_SKL_TrpC_r. The base-triplets encoding the SKL-motif is contained in the overhang of primer Neo_SKL_TrpC_r and was adapted to the codon usage of $S$. macrospora according to the HIVE-Codon Usage Table (https://hive.biochemist ry.gwu.edu/review/codon2) (Athey et al. 2017). To put the $m n g-S K L$ gene under control of the constitutive gpd promoter and $\operatorname{trp} C$ terminator of $A$. nidulans, we amplified the promoter and terminator from plasmid p1783-1 with primer pair pRS_Pgpd_f/Pgpd_Neo_r and TtrpC_f / TtrpC_pRS_r, respectively. The three PCR products were cloned into XhoI-linearized vector pRS-nat by homologous recombination in yeast strain PJ69-4A (Colot et al. 2006). The resulting plasmid was designated pmng-SKL (Fig. 1c). DNA sequencing of the plasmids was performed by Seqlab Sequence Service Laboratories $\mathrm{GmbH}$ (Göttingen, Germany).

\section{Generation of S. macrospora strains}

All strains used in this study are listed in Additional file 1: Table S1. Strains were generated by transforming the plasmids (pxyl-mng, pGG-C-F-mng, pmng-SKL) 

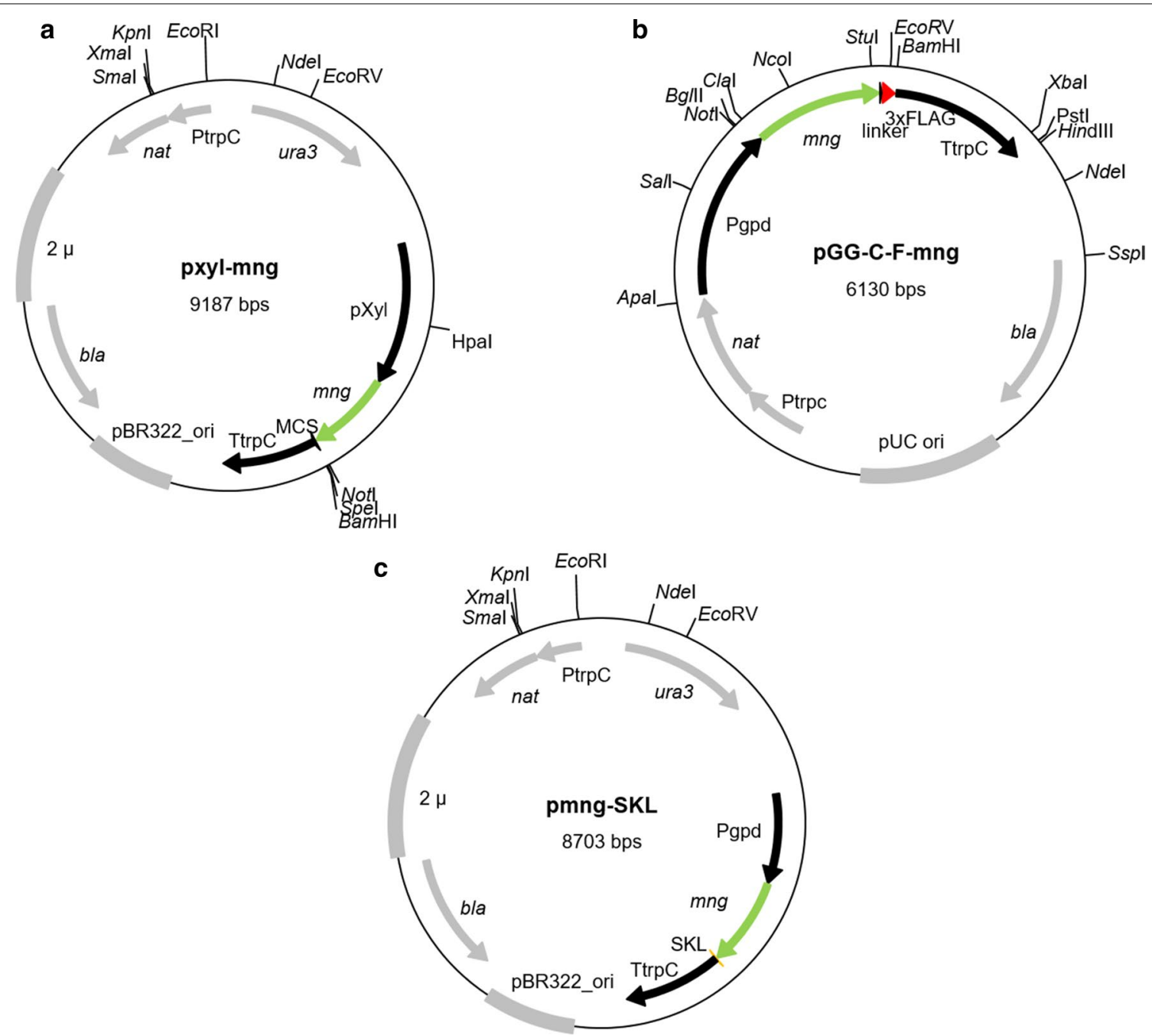

Fig. 1 Physical maps of mNG plasmids. a pxyl-mng, b pGG-C-F-mng, c pmng-SKL The ampicillin resistance cassette (b/a) and the ori for selection and replication in E. coli are indicated. The nourseothricin resistance gene nat 1 is under control of the A. nidulans trpC promoter (PtrpC) can be used for selection of fungal transformants. The $2 \mu$ ori and the ura3 gene can be used for transformation of S. cerevisiae. The mng ORF (green arrow) is either regulated by the xylose inducible Smxyl promoter of S. macrospora (Pxyl) or the gpd promoter A. nidulans (Pgpd) and the trpC terminator of A. nidulans (TtrpC). Plasmid pxyl-mng contains a multiple cloning site (MCS). In plasmid pGG-C-F-mng a C-terminal $3 \times$ FLAG tag: (DYKDHD-G-DYKDHD-I-DYKDDDDK, red arrow) is fused via a short linker (GSGSG) to mNG. In plasmid pmng-SKL the peroxisomal targeting sequences SKL (orange arrow) is fused to the C-terminus of $\mathrm{mNG}$

presented in Table 2 into the S. macrospora wild type strain. Strains with ectopically integrated fusion constructs were selected on BMM medium supplemented with nourseothricin-dihydrogen sulphate (Hoff and Kück 2006; Walz and Kück 1995). Primary transformants were crossed with the color spore mutant fus1-1 (Nowrousian et al. 2012) to obtain single spore isolates (ssi) as described previously (Bernhards and Pöggeler, 2011). At least two independent ssi were analyzed in fluorescent microscopy.

\section{Protein sample preparation and Western-blot analysis}

$S$. macrospora strains were cultivated in corresponding media for three days. For the protein preparation, mycelium was harvested, dried, and ground in liquid nitrogen. $1 \mathrm{~g}$ of mycelium was combined with $560 \mu \mathrm{l}$ extraction buffer (100 mM Tris- $\mathrm{HCl} \mathrm{pH}$ 7.5, $150 \mathrm{mM} \mathrm{NaCl}, 2 \mathrm{mM}$ EDTA) with freshly added $2 \mathrm{mM}$ DTT, $1 \mathrm{mM}$ PMSF, $0.2 \%$ NP-40, protease inhibitor cocktail IV (1tbl/50 ml, 04693132001, Mannheim, Germany) and PhosSTOP ${ }^{\mathrm{TM}}$ (1 tbl/10 ml, Roche, 04906837001, Mannheim, Germany). Protein samples were centrifuged for $20 \mathrm{~min}$ at 12,000 rpm and $4{ }^{\circ} \mathrm{C}$. Afterwards, $45 \mu \mathrm{l}$ of the supernatant was boiled with $50 \mu \mathrm{l}$ of $2 \times$ Tris-glycerine/ SDS-sample buffer (Serva, 
P190078, Heidelberg, Germany) and $5 \mu \mathrm{l}$ of $1 \mathrm{M}$ DTT for $2 \mathrm{~min}$ at $85^{\circ} \mathrm{C}$. $15 \mu \mathrm{l}$ of the samples were loaded on a $12 \%$ SDS gel, together with the Nippon Genetics Co. Europe blue star pre-stained protein marker (NIPPON Genetics Europe, MWP03, Düren, Germany). Proteins separated in SDS-PAGE were transferred to a AmershamTM ProtranTM Nitrocellulose Blotting Membrane (GE Healthcare, RPN203B, Little Chalfont, UK) applying $300 \mathrm{~mA}$ a semi-dry device (Biometra, Fastblot 014-200 type B34, Göttingen, Germany) (Towbin et al. 1979). Transfer of proteins to the membrane was visualized by staining the membrane with Ponceau red solution (3\% TCA, 0.2\% Ponceau S) for 45 min and washing with A.dest.

Nitrocellulose membranes containing transferred proteins were washed once with $1 \times$ Tris-buffered saline supplemented with $0.05 \%$ Tween $20^{\circledR}$ (TBST) for $15 \mathrm{~min}$ and blocked with $5 \%(\mathrm{w} / \mathrm{v})$ milk powder in TBST for $1 \mathrm{~h}$ at room temperature. Antigen-antibody reaction was performed using a mNeonGreen-antibody (1:5000; ChromoTek GmbH, 32f6-20, Planegg-Martinsried, Germany) and a horse-radish peroxidase (HRP) coupled secondary anti mouse-antibody (1:10,000 Sigma-Aldrich, A5278) solved in 5\% skim milk/TBST. The membrane and the primary antibody solution were incubated over night at $4{ }^{\circ} \mathrm{C}$. After the antibody was removed, the membrane was washed three times for $15 \mathrm{~min}$ with TBST. The secondary antibody was applied to the membrane for $1 \mathrm{~h}$ at room temperature and the membrane was washed three times with TBST. Enhanced chemiluminescent reaction was used to detect the HRP coupled antibodies using the ImmobilonTM Western HRP Substrate kit (Merck, WBKLS0500, Kenilworth, New Jersey, USA).

\section{Microscopy}

To ensure that fungal cultures of identical developmental stages were compared in light and fluorescence microscopy, strains were grown for $24 \mathrm{~h}$ at $27^{\circ} \mathrm{C}$ on BMM or SWG medium with cellophane and investigated with the AxioImager M1 microscope (Zeiss, Jena, Germany) (Rech et al. 2007). Microspcopic analysis was performed with at least two independent transformats in at least three replicates. Images were captured using a Photometrix CoolSNAP HQ camera (Roper Scientific, Photometrics, Tucson, AZ, USA) and image processing was done using ZEISS ZEN Digital Imaging (version 2.3; Zeiss): Exposure time was identical for all images. For the detection of the eGFP and mNG signals Chroma filter set 49002 (exciter ET470/40x, ET525/50 m, beamsplitter T495lpxr) and for DsRed Chroma filter set 49005 (exciter ET545/30x, emitter ET620/60 $\mathrm{m}$ and beamsplitter T570LP) were used. In addition, we used YFP Chroma filter set 49003 (exciter ET500/20x, emitter ET535/30 m and beamsplitter T515LP) for fluorescent mNG signals.

\section{Results}

Construction of fungal $\mathrm{mNeonGreen}$ reporter plasmids

The visualization of fluorescent reporter proteins is an important technique in fungal cell biology. Here, we examined the utilization of the lancelet yellow-green fluorescent protein for fluorescent microscopy in the filamentous ascomycete $S$. macrospora. From our experience, codon-usage optimized human genes encoding for fluorescent proteins perform best in S. macrospora (Pöggeler et al. 2003). Therefore, we employed a human codon-optimized version of the mNG gene, kindly provided by Dr. Kay Oliver Schink (Centre for Cancer Biomedicine (CCB) Oslo University Hospital, Radiumhospitalet). This $m n g$ version harbors 35 silent base mutations to be human codon-optimized for high expression levels in human cells (Additional file 1: Figure S1). In comparison to the original sequence [KC295282.1, (Shaner et al. 2013)], none of the four least used codons of $S$. macrospora are present in this human codon-optimized mng gene (Athey et al. 2017). Furthermore, with a $55.84 \%$ GC-content the humanized $m n g$ version is very similar to the $56.5 \% \mathrm{GC}$-content of coding regions in $S$. macrospora (Nowrousian et al. 2012).

To avoid a putative toxicity of $\mathrm{mNG}$ we first cloned $m n g$ under control of the xylose inducible promoter Smxyl of $S$. macrospora in plasmid pxyl-mng (Bloemendal et al. 2014) (Fig. 1a). The vector contains three unique sites of the commonly used restriction enzymes NotI, SpeI, and BamHI. All sites are located downstream of the $m n g$ gene and can be employed to C-terminally fuse fungal genes of interest. In plasmid pGG-C-F-mng, mng is under control of the constitutive A. nidulans gpd promoter and is further fused to a $3 \times$ FLAG Tag, which can be used for Western-blot experiments with a commercially available FLAG antibody (Fig. 1b). Further, we constructed a mNG-SKL plasmid for labelling of peroxisomes, carrying $m n g-S K L$ under control of the constitutive gpd promoter as well (Fig. 1c).

\section{Expression of $\mathrm{mNG}$ in S. macrospora}

First, we applied plasmid pxyl-mng for transformation of $S$. macrospora wild type. Since this plasmid encodes mNG under control of the xylose inducible Smxyl promoter, we tested expression of the $m n g$ gene under different growth conditions by means of fluorescence microscopy and Western-blot analysis. Cultures were grown in synthetic Westergaards (SWG) medium (Nowrousian et al. 2005) supplemented with either glucose or xylose as the sole carbon source or on complex corn meal medium (BMM) (Towbin et al. 1979). In fluorescence microscopy, under all growth conditions the yellow green fluorescence signal was detectable in the cytoplasm of the transformants. The signal was clearly 
excluded from the vacuoles. The strongest signal could be observed under inducing conditions (SWG + xylose), whereas a weaker signal was detectable under repressed conditions (SWG+glucose) and growth in the complex BMM medium (Fig. 2).

These results were verified by Western-blot analysis (Fig. 3). After growth for 3 days in the respected liquid medium, mycelium was harvested and proteins were extracted from the transformants for SDS-PAGE and Western-blot analysis with an
anti-mNeonGreen-antibody. We used transformants expressing eGFP as a negative control. A clear $\mathrm{mNG}$ signal of around $27 \mathrm{kDa}$ was detectable for all three conditions. The strongest signal was visible under inducing conditions (SWG + xylose). In all lanes, a second band at slightly higher molecular weight than expected can be observed. This may be due to glycosylation or other protein modifications. $\mathrm{mNG}$ has only very little sequence identity in common with eGFP (Stoddard and Rolland 2019), therefore no cross reactivity of the mNeonGreen

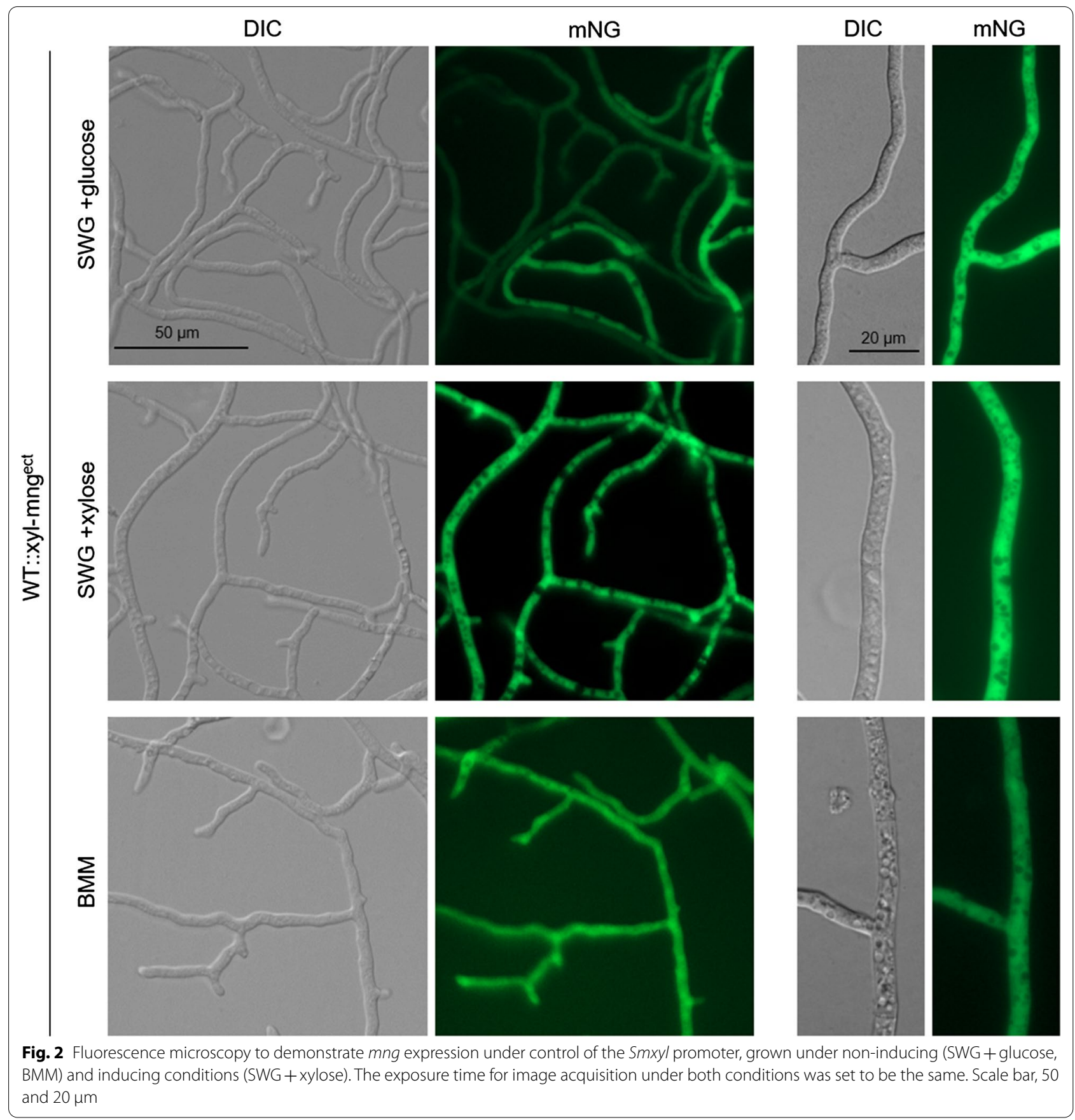




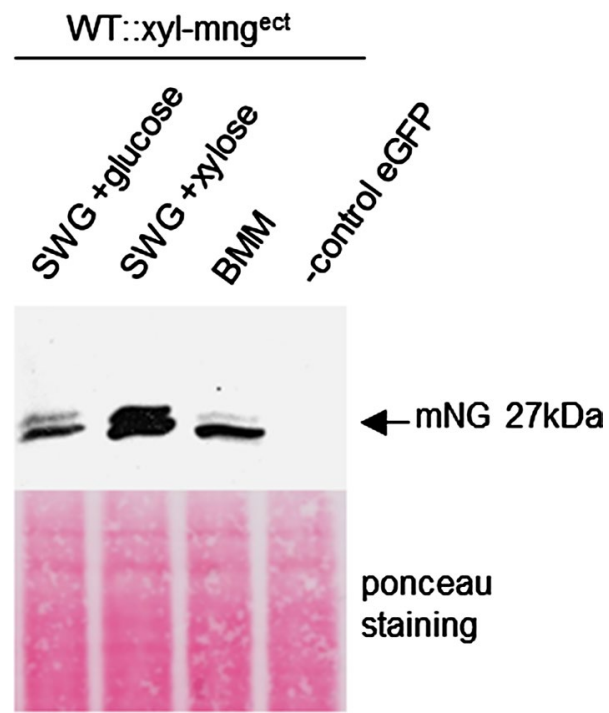

Fig. 3 Western-blot analysis of total protein extracts to demonstrate mng expression under control of the $\mathrm{Smxyl}$ promoter grown under non-inducing (SWG + glucose) and inducing conditions (SWG + xylose), as well as in BMM. An S. macrospora wt transformant carrying plasmid p1783 and expressing free eGFP served as a control. Ponceau red staining of the blot before the immunodetection served as loading control

antibody was visible with eGFP (Fig. 3). These data indicate that the human codon optimized version of the $m n g$ gene can be expressed under control the inducible $S m x y l$ promoter.

Next, we tested the expression of the $m n g$ gene under control of the strong constitutive gpd promoter of $A$. nidulans. Western-blot analysis with the mNeonGreen antibody revealed a strong signal (Additional file 1: Figure S2). Compared to strains expressing DsRed or eGFP under control of the gpd promoter the fluorescence signal of $\mathrm{mNG}$ appeared almost equal (Fig. 4). The fluorescence intensity of strains expressing $m n g$ under control of the constitutive gpd promoter is similar to strains expressing mng under control of the inducible $S m x y l$ promoter upon growth under inducing conditions (Fig. 4a, b). In all strains, the fluorescence was uniformly visible throughout the cytoplasm of the hyphae and appeared to be excluded from the vacuoles.

In comparison to EGFP (excitation 488, emission 507), mNG has a yellow-shifted excitation maximum of $506 \mathrm{~nm}$ and an emission maximum of $517 \mathrm{~nm}$, which is more similar to the yellow fluorescent protein EYFP (excitation 514 $\mathrm{nm}$ and emission 527). Shaner et al. (2013) recommended to image mNG using standard green fluorescent protein band-pass or long-pass filter sets, or with yellow fluorescent protein filter sets having only minimal reduction in collection efficiency. Therefore, we tested in addition to eGFP the eYFP filter set for mNG fluorescent microscopy. In comparison to the standard eGFP filter set, the usage of the eYFP filter lead to no obvious increase in fluorescence or brightness, only the contrast seems to be increased (Additional file 1: Figure S3). Furthermore, mNG revealed no fluorescence signal with the DsRed filter set (Additional file 1: Figure S3). Therefore, co-localization studies with red fluorescent proteins such as DsRed, mRFP, TagRFP-T, and tdTomato might be applicable in combination with $\mathrm{mNG}$.

To demonstrate that $\mathrm{mNG}$ is suitable to label fungal peroxisomes, we fused the peroxisomal PTS1 signal SKL to the C-terminus of $\mathrm{mNG}$ and transformed plasmid pmngSKL into the S. macrospora wild-type strain. Fluorescence microscopy of transformants carrying pmng-SKL led to a punctate fluorescent pattern (Fig. 5a). Similarly, a SKLtagged DsRed previously used for peroxisomal studies in $S$. macrospora exhibited a punctate peroxisomal fluorescence signal (Fig. 5b) (Elleuche and Pöggeler 2008).

\section{Discussion}

Important innovations in fluorescent protein performance have been described with the discovery of brighter, more readily maturing and more photostable fluorescent proteins (Botman et al. 2019; Heppert et al. 2016). However, often these new proteins have not yet been tested with regard to their potential in filamentous fungi. Here, we expressed the yellow-green fluorescent protein mNG for the first time in S. macrospora. By using an inducible and a constitutive promoter, we generated transformants emitting bright and stable green fluorescence. Furthermore, we accomplished visualization of the peroxisomes by tagging $\mathrm{mNG}$ with a peroxisomal signal peptide.

The yellow-green fluorescent protein $\mathrm{mNG}$ was predicted to be superior to eGFP with regard to its brightness and photostability (Shaner et al. 2013), however, these effects were not that evident when the mng gene was expressed in S. macrospora. In vivo, mNG performed very much alike to eGFP. A similar observation has been described in the nematode Caenorhabditis elegans where both fluorescent proteins behaved similarly in most experiments and $\mathrm{mNG}$ was only a better tag than eGFP in specific kinds of experiments (Heppert et al. 2016). In Dictyostelium discoideum mNG showed an increased brightness in comparison to eGFP (Paschke et al. 2018). Similarly, when using the EYFP filter set instead of the EGFP filter set, the fluorescence of $\mathrm{mNG}$ appeared brighter in S. macrospora. Therefore, one has to ensure to adapt filter set to $\mathrm{mNG}$ fluorescence.

In the yeast $S$. cerevisiae, a recent comprehensive in vivo characterization of 27 fluorescent proteins also revealed that in vitro properties of fluorescent proteins often only 


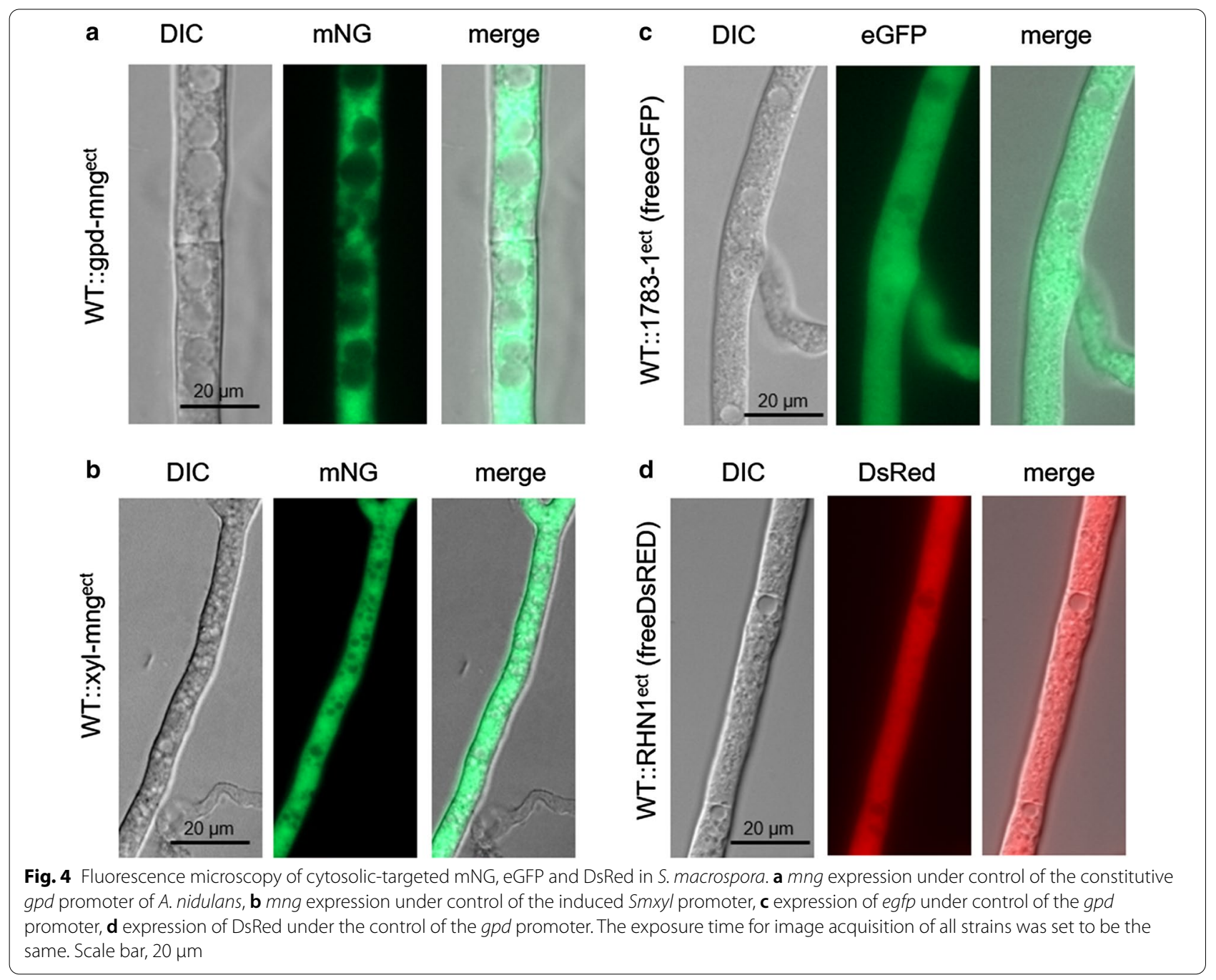

poorly reflect their in vivo performance. However, in yeast mNG was among the most photostable fluorescent proteins (Botman et al. 2019). Moreover, in Nicotiana benthamiana and S. cerevisiae, mNG was shown to be one of the best performing green fluorescent proteins under dynamic pH conditions (Botman et al. 2019; Stoddard and Rolland 2019). These conditions were not tested in our study.

For S. macrospora, we used a human codon usage optimized version of $m n g$, confirming our previous experience with the expression of a human codon-optimized version of egfp (Pöggeler et al. 2003). It is quite possible that a specific $S$. macrospora codon-optimized version of $m n g$ and/or the addition of synthetic introns will perform even better, as it was shown for modified $m n g$ versions in human, C. elegans and S. cerevisiae (Botman et al. 2019; Heppert et al. 2016; Tanida-Miyake et al. 2018).

The selection of a suitable promoter is critical for the expression of heterologous fluorescent proteins in filamentous fungi. We have chosen the constitutive $A$. nidulans gpd promoter successfully applied in a variety of fungal species and additionally the inducible $S$. macrospora $S m x y l$ promoter, previously reported to be functional in Acremonium chrysogenum (Bloemendal et al. 2014; Zhang et al. 2020). Therefore, the expression plasmids presented here are likely useful for the expression of $m n g$ in filamentous fungi other than S. macrospora.

Labelling of peroxisomes with reporter fluorescent proteins carrying signal peptides for peroxisomal import is a powerful method for investigating peroxisome dynamics by means of live cell imaging. We showed that mNG-SKL efficiently labels peroxisomes in $S$. macrospora, similar to other fluorescent proteins like eGFP and DsRed carrying a C-terminal SKL tripeptide for peroxisomal matrix import (Elleuche and Pöggeler 2008; Idnurm et al. 2007; Ruprich-Robert et al. 2002).

In conclusion, the vectors for expression of $m n g$ and labelling of peroxisomes presented here will provide an alternative for tagging of proteins for fungal cell biology 


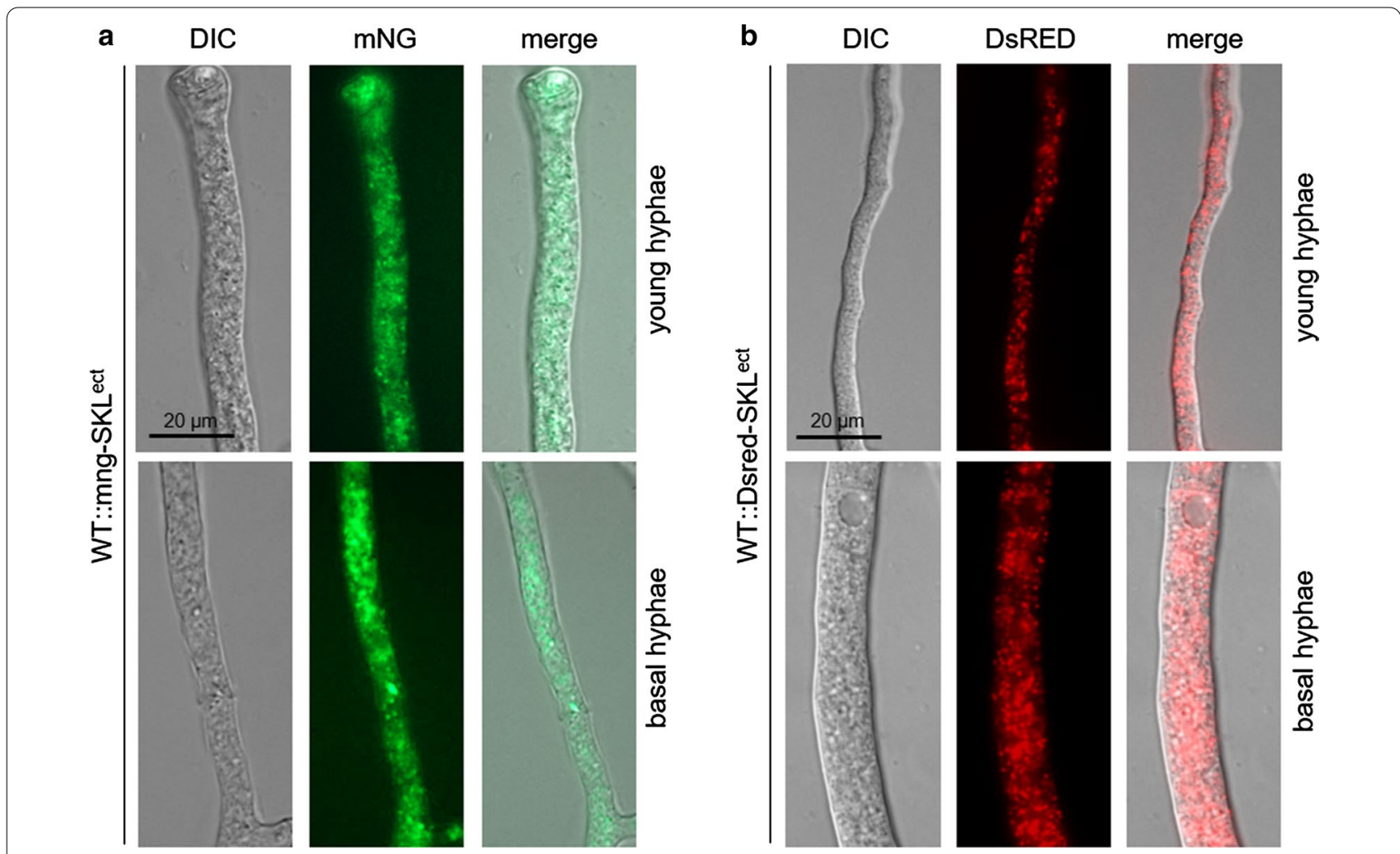

Fig. 5 Fluorescence microscopy of peroxisomal-targeted mNG and DsRed in S. macrospora. a Hyphae of a strain transformed with pmng-SKL. b Hyphae of a strain transformed with pDsRed-SKL. The exposure time for image acquisition of both strains was set to be the same. Scale bar, $20 \mu \mathrm{m}$

and biochemistry. Although the plasmids were primarily constructed for $S$. macrospora they should prove very effective in a wide variety of filamentous as well.

\section{Supplementary Information}

The online version contains supplementary material available at https://doi. org/10.1186/s13568-020-01160-x.

Additional file 1: Table S1. Overview of strains used and constructed in this study. Figure S1. Lalign (https://embnet.vital-it.ch/software/LALIG N_form.html) alignment of the human codon-optimized mNG version (mNGhco) and the mNG sequence published by (Shaner et al. 2013). Figure S2. Western-blot analysis with a NeonGreen antibody of the total protein extracts from a transformant carrying plasmid pGG-C-F-mng to demonstrate $m n g$ expression under control of the gpd promoter. Figure S3. Fluorescence microscopy of cytosolic-targeted mNG in S. macrospora wt strain under control of the constitutive gpd promoter of $A$. nidulans. Fluorescence was recorded with different filter sets: eGFP (Chroma filter set 49002) eYFP (Chroma filter set 49003), DsRed (Chroma filter set 49005). The exposure time for image acquisition of all strains was set to be the same. Scale bar, $50 \mu \mathrm{m}$

\section{Acknowledgements}

We gratefully acknowledge Dr. Kay Oliver Schink, Centre for Cancer Biomedicine (CCB) Oslo University Hospital, Radiumhospitalet for kindly providing mNG vector 753-pENTR20 mNeonGreen-N1 and Dr. Ines Teichert (RuhrUniversity Bochum, Germany) for sharing plasmids for the GoldenGate cloning system. We wish to thank Dr. Daniela Nordzieke for critically reading the manuscript.

\section{Authors' contributions}

AW and SP made the experimental design; AW, KO, GS performed experiments and analyzed data; AW and SP wrote the manuscript. All authors read and approved the final manuscript.

\section{Funding}

Open Access funding enabled and organized by Projekt DEAL. This work was funded by the Deutsche Forschungsgemeinschaft PO 523/8-1.

\section{Availability of data and materials}

All vectors described are available on request.

\section{Ethics approval and consent to participate}

Not applicable.

\section{Consent for publication}

All authors give their consent for publication.

\section{Competing interests}

All authors declare no competing interests.

Received: 22 October 2020 Accepted: 7 December 2020

Published online: 21 December 2020

\section{References}

Athey J, Alexaki A, Osipova E, Rostovtsev A, Santana-Quintero LV, Katneni U, Simonyan V, Kimchi-Sarfaty C (2017) A new and updated resource for codon usage tables. BMC Bioinform 18:391 
Bagar T, Altenbach K, Read ND, Bencina M (2009) Live-Cell imaging and measurement of intracellular $\mathrm{pH}$ in filamentous fungi using a genetically encoded ratiometric probe. Eukaryot Cell 8:703-712

Berepiki A, Lichius A, Shoji J-Y, Tilsner J, Read ND (2010) F-Actin dynamics in Neurospora crassa. Eukaryot Cell 9:547-557

Bernhards Y, Pöggeler S (2011) The phocein homologue SmMOB3 is essential for vegetative cell fusion and sexual development in the filamentous ascomycete Sordaria macrospora. Curr Genet 57:133-149

Bloemendal S, Löper D, Terfehr D, Kopke K, Kluge J, Teichert I, Kück U (2014) Tools for advanced and targeted genetic manipulation of the $\beta$-lactam antibiotic producer Acremonium chrysogenum. J Biotechnol 169:51-62

Botman D, de Groot DH, Schmidt P, Goedhart J, Teusink B (2019) In vivo characterisation of fluorescent proteins in budding yeast. Sci Rep 9:2234

Campbell RE, Tour O, Palmer AE, Steinbach PA, Baird GS, Zacharias DA, Tsien RY (2002) A monomeric red fluorescent protein. Proc Natl Acad Sci USA 99:7877-7882

Chalfie M, Tu Y, Euskirchen G, Ward W, Prasher D (1994) Green fluorescent protein as a marker for gene expression. Science 263:802-805

Chapuis AF, Ballou ER, MacCallum DM (2019) A Bright future for fluorescence imaging of fungi in living hosts. J Fungi 5:29

Chudakov DM, Lukyanov S, Lukyanov KA (2005) Fluorescent proteins as a toolkit for in vivo imaging. Trends Biotechnol 23:605-613

Colot HV, Park G, Turner GE, Ringelberg C, Crew CM, Litvinkova L, Weiss RL, Borkovich KA, Dunlap JC (2006) A high-throughput gene knockout procedure for Neurospora reveals functions for multiple transcription factors. Proc Natl Acad Sci USA 103:10352-10357

Dahlmann T, Terfehr D, Becker K, Teichert I (2020) Golden Gate vectors for efficient gene fusion and gene deletion in diverse filamentous fungi. Curr Genet. https://doi.org/10.1007/s00294-020-01143-2

Elleuche S, Pöggeler S (2008) Visualization of peroxisomes via SKL-tagged DsRed protein in Sordaria macrospora. Fungal Genet Rep 55:8-12

Esser K (1982) Cryptogams - cyanobacteria, algae, fungi, lichens. Cambridge University Press, London

Heppert JK, Dickinson DJ, Pani AM, Higgins CD, Steward A, Ahringer J, Kuhn JR, Goldstein B (2016) Comparative assessment of fluorescent proteins for in vivo imaging in an animal model system. Mol Biol Cell 27:3385-3394

Hoff B, Kück U (2005) Use of bimolecular fluorescence complementation to demonstrate transcription factor interaction in nuclei of living cells from the filamentous fungus Acremonium chrysogenum. Curr Genet 47:132-138

Hoff B, Kück U (2006) Application of the nourseothricin acetyltransferase gene (nat1) as dominant marker for the transformation of filamentous fungi. Fungal Genet Rep 53:9-11

Hostettler L, Grundy L, Käser-Pébernard S, Wicky C, Schafer WR, Glauser DA (2017) The bright fluorescent protein $\mathrm{mNeonGreen} \mathrm{facilitates} \mathrm{protein}$ expression analysis in vivo. G3 7:607-615

Idnurm A, Giles SS, Perfect JR, Heitman J (2007) Peroxisome function regulates growth on glucose in the basidiomycete fungus Cryptococcus neoformans. Eukaryot Cell 6:60-72

James P, Halladay J, Craig EA (1996) Genomic libraries and a host strain designed for highly efficient two-hybrid selection in yeast. Genetics 144:1425-1436

Janus D, Hoff B, Hofmann E, Kück U (2007) An efficient fungal RNA-silencing system using the DsRed reporter gene. Appl Environ Microbiol 73:962-970

Klix V, Nowrousian M, Ringelberg C, Loros JJ, Dunlap JC, Pöggeler S (2010) Functional characterization of MAT1-1-specific mating-type genes in the homothallic ascomycete Sordaria macrospora provides new insights into essential and nonessential sexual regulators. Eukaryot Cell 9:894-905

Lorang JM, Tuori RP, Martinez JP, Sawyer TL, Redman RS, Rollins JA, Wolpert TJ, Johnson KB, Rodriguez RJ, Dickman MB, Ciuffetti LM (2001) Green fluorescent protein is lighting up fungal biology. Appl Environ Microbiol 67:1987-1994

Mikkelsen L, Sarrocco S, Lübeck M, Jensen DF (2003) Expression of the red fluorescent protein DsRed-Express in filamentous ascomycete fungi. FEMS Microbiol Lett 223:135-139

Nowrousian M, Ringelberg C, Dunlap JC, Loros JJ, Kück U (2005) Cross-species microarray hybridization to identify developmentally regulated genes in the filamentous fungus Sordaria macrospora. Mol Genet Genom 273:137-149

Nowrousian M, Teichert I, Masloff S, Kück U (2012) Whole-genome sequencing of Sordaria macrospora mutants identifies developmental genes. G3 2:261-270
Oda K, Terado S, Toyoura R, Fukuda H, Kawauchi M, Iwashita K (2016) Development of a promoter shutoff system in Aspergillus oryzae using a sorbitolsensitive promoter. Biosci Biotechnol Biochem 80:1792-1801

Paschke P, Knecht DA, Silale A, Traynor D, Williams TD, Thomason PA, Insall RH, Chubb JR, Kay RR, Veltman DM (2018) Rapid and efficient genetic engineering of both wild type and axenic strains of Dictyostelium discoideum. PLOS ONE 13:e0196809

Pham T, Xie X, Lin X (2020) An intergenic "safe haven" region in Aspergillus fumigatus. Med Mycol 58:1178-1186

Pöggeler S, Masloff S, Hoff B, Mayrhofer S, Kück U (2003) Versatile EGFP reporter plasmids for cellular localization of recombinant gene products in filamentous fungi. Curr Genet 43:54-61

Rech C, Engh I, Kück U (2007) Detection of hyphal fusion in filamentous fungi using differently fluorescence-labeled histones. Curr Genet 52:259-266

Ruprich-Robert G, Berteaux-Lecellier V, Zickler D, Panvier-Adoutte A, Picard M (2002) Identification of six loci in which mutations partially restore peroxisome biogenesis and/or alleviate the metabolic defect of pex 2 mutants in Podospora. Genetics 161:1089-1099

Sambrook J, Fritsch E, Maniatis T (2001) Molecular cloning: a laboratory manual. Cold Spring Harbor Laboratory Press, Cold Spring Harbor

Schuster M, Kilaru S, Guo M, Sommerauer M, Lin C, Steinberg G (2015) Red fluorescent proteins for imaging Zymoseptoria tritici during invasion of wheat. Fungal Genet Biol 79:132-140

Shaner NC, Lambert GG, Chammas A, Ni Y, Cranfill PJ, Baird MA, Sell BR, Allen JR, Day RN, Israelsson M, Davidson MW, Wang J (2013) A bright monomeric green fluorescent protein derived from Branchiostoma lanceolatum. Nat Methods 10:407-409

Stoddard A, Rolland V (2019) I see the light! Fluorescent proteins suitable for cell wall/apoplast targeting in Nicotiana benthamiana leaves. Plant Direct 3:e00112

Tanida-Miyake E, Koike M, Uchiyama Y, Tanida I (2018) Optimization of mNeonGreen for Homo sapiens increases its fluorescent intensity in mammalian cells. PLOS ONE 13:e0191108

Teichert I, Nowrousian M, Pöggeler S, Kück U (2014) The filamentous fungus Sordaria macrospora as a genetic model to study fruiting body development. Adv Genet 87:199-244

Teichert I, Pöggeler S, Nowrousian M (2020) Sordaria macrospora: 25 years as a model organism for studying the molecular mechanisms of fruiting body development. Appl Microbiol Biotechnol 104:3691-3704

Terfrüchte M, Joehnk B, Fajardo-Somera R, Braus GH, Riquelme M, Schipper K, Feldbrügge M (2014) Establishing a versatile Golden Gate cloning system for genetic engineering in fungi. Fungal Genet Biol 62:1-10

Toews MW, Warmbold J, Konzack S, Rischitor P, Veith D, Vienken K, Vinuesa C, Wei H, Fischer R (2004) Establishment of mRFP1 as a fluorescent marker in Aspergillus nidulans and construction of expression vectors for highthroughput protein tagging using recombination in vitro (GATEWAY). Curr Genet 45:383-389

Towbin H, Staehelin T, Gordon J (1979) Electrophoretic transfer of proteins from polyacrylamide gels to nitrocellulose sheets: procedure and some applications. Proc Natl Acad Sci USA 76:4350-4354

Walz M, Kück U (1995) Transformation of Sordaria macrospora to hygromycin B resistance: characterization of transformants by electrophoretic karyotyping and tetrad analysis. Curr Genet 29:88-95

Wilton R, Ahrendt AJ, Shinde S, Sholto-Douglas DJ, Johnson JL, Brennan MB, Kemner KM (2017) A new suite of plasmid vectors for fluorescence-based imaging of root colonizing pseudomonads. Front Plant Sci 8:2242

Xiao C, Li L, Lao L, Liu Y, Wei Q, Ji Q, Sun G, Lin F, Wang J, Bao G (2018) Application of the red fluorescent protein mCherry in mycelial labeling and organelle tracing in the dermatophyte Trichophyton mentagrophytes. FEMS Microbiol Lett 365:1-9

Zhang T, Liu H, Lv B, Li C (2020) Regulating strategies for producing carbohydrate active enzymes by filamentous fungal cell factories. Front Bioeng Biotechnol 8:691

Zickler D, Espagne E (2016) Sordaria, a model system to uncover links between meiotic pairing and recombination. Semin Cell Dev Biol 54:149-157

\section{Publisher's Note}

Springer Nature remains neutral with regard to jurisdictional claims in published maps and institutional affiliations. 